\title{
P04-1-17 Poster session
}

\section{Pharmacological study of new compounds - the translocator protein 18kDa ligands}

\author{
Milada Yarkova, Grigoriy Mokrov, Tatyana Gudasheva, Sergey Seredenin \\ Zakusov Institute of Pharmacology, Russia
}

Translocator protein $18 \mathrm{kDa}$ (TSPO) is considered as a target for creating effective anxiolytics. This transporter provides cholesterol transport from the outer to the inner mitochondrial membrane that is the rate-limiting step of neurosteroids biosynthesis. Neurosteroids are potent positive allosteric modulators of GABAA receptor, which plays an important role in the pathophysiology of anxiety disorders. Based on the pharmacophore model of the structures of TSPO ligands and using the molecular docking method, we constructed the molecules of GML-1 (N-benzyl-N-methyl-1-phenylpyrrolo[1,2-a] pyrazine-3-carboxamide) and GML-3 (N-butyl-N-methyl-1-phenylpyrrolo[1,2-a]pyrazin-3-carboxamide).

Methods: radioligand binding assay, the "elevated plus-maze" test, the conflict Vogel test, the tail suspension test, the novel object recognition test

Results: The interaction of new original 1-arylpyrrolo[1,2-a]pyrazine-3-carboxamides derivatives with TSPO was studied by radioligand binding assay. Compounds GML-1 ( $\mathrm{Ki}=5,2 \times 10-8 \mathrm{M})$ and GML-3 ( $\mathrm{Ki}=5,3 \times 10-7 \mathrm{M})$ have high binding affinity for TSPO. GML-1 and GML-3 in the dose range of 0.1-5.0 mg/kg, i.p. and per os in the standard psychopharmacological tests in ICR mice and Wistar rats demonstrated the anxiolytic-like effects, that were blocked by the TSPO selective antagonist PK11195. Anxiolytic effect of GML-1 and GML-3 was also completely blocked by neurosteroidogenesis enzyme inhibitors trilostane and finasteride.

It was established that the compound GML-3 in a dose range $0,5-5,0 \mathrm{mg} / \mathrm{kg}$, i.p. and five-day injection in a dose 0,5 $\mathrm{mg} / \mathrm{kg}$, i.p. displays antidepressant effect in a tail suspension test. With the use of specific TSPO antagonist - PK11195 it was determined that antidepressant effect of GML 3 depends on binding to TSPO receptor site. The compound GML-1 possesses antidepressant effect in the same test after five-day injection in a dose of $0,5 \mathrm{mg} / \mathrm{kg}$, i.p. GML-1 in a dose range of $0,05-0,5 \mathrm{mg} / \mathrm{kg}$ displays nootropic effect in rodents.

GML-1 and GML-3 have a low acute toxicity (LD50 > $1000 \mathrm{mg} / \mathrm{kg}$ ), and they well penetrates the BBB. The tableted dosage form of GML-1 has been developed.

Conclusions: The data obtained on the molecular target, the spectrum of psychotropic activity and low toxicity suggest that GML-1 and GML-3 are promising drugs for development as a medicine targeted for the treatment of anxiety and depressive disorders. 